\title{
Special Section Guest Editorial
}

Near-infrared spectroscopy (NIRS) has been used for noninvasive monitoring of changes in tissue oxygen levels since the late 1970s and gathered a world-wide interest because of its wide-range applicability to both research and clinics. Such measurements rely on oxygen-dependent absorption changes that occur in the heme- and copper-containing compounds of tissue and on an adequate penetration into biological tissue. Continuous-wave NIRS is rather small and portable, therefore suitable to clinical research. However, tissue oxygenation measured by this method is only qualitative in nature because of unquantifiable biophysical quantities such as the optical pathlength. Time and frequency domain NIRS provide accurate values of scattering and absorption and the calculated oxy- and deoxy-hemoglobin concentration and hemoglobin saturation. Another problem we have to address in oxygenation measurement by NIRS is the heterogeneity of the sampling tissue. This will only be solved by using multiple source-detector arrays to determine the spatial heterogeneity of tissue oxygenation. Recently, imaging technology has begun to be developed and has provided important knowledge to basic science and clinical application in the study of tissue oxygenation and functional activation of brain and muscle.

In viewing the trend in this research area, we founded the Japanese Society on Medical Near Infrared Spectroscopy in 1994 and held annual national scientific meetings since then. Last year, we organized the First International Symposium on Japanese Medical Near Infrared Spectroscopy in Tokyo and accommodated 57 papers including 9 invited lecturers. The papers presented in the symposium covered a variety of subjects in NIRS research, including technical and theoretical aspects, clinical application in neurology, neurosurgery, anesthesiology, and vascular surgery, muscle and nerve diseases, and muscle physiology and sports medicine. We had published the proceedings of the four consecutive annual meetings, primarily in Japanese. We have decided this time to submit the proceedings for publication in an international journal to disclose the outcomes from the Japanese Society to the outside world instead of hiding them by publishing in Japanese, as had been suggested by the researchers overseas.

This special section consists of two review articles by Jöbsis-vanderVliet et al. with regard to some of the most important early research for initiating NIRS study in medicine, two papers by Koizumi et al. and Yamashita et al. regarding brain function analysis by dynamic optical imaging, and two papers dealing with perspectives on the clinical research in neuropharmacology by Yamaguchi et al. and in gastroenterology by Nishio et al.

The special section contains the first collection of the studies presented in the First International Symposium on Japanese Medical Near Infrared Spectroscopy and reviewed, revised, and accepted for publication as original articles in JBO. More papers presented in the symposium are planned for publication in later issues of the journal.

Kazuo Okada, Teikyo University Takafumi Hamaoka, Tokyo University Special Section Guest Editors 